fields emit ultraviolet radiation that energizes the planet's atmosphere.

Spake et al. observed WASP-107b using a camera on board the Hubble Space Telescope, and concluded that the planet's atmosphere escapes to form a comet-like tail (Fig. 1). Astronomers have long known that giant planets can lose their atmospheres in this fashion ${ }^{5}$, so this aspect of Spake and colleagues' work is not surprising. But the authors have added a key twist to the story. Until now, only hydrogen (the main component of giant planets) and a few elements with low abundances ${ }^{6}$ have been identified in eroding exoplanetary atmospheres.

Atoms in the gaseous tail of an exoplanet are most easily detected when they absorb stellar light during a transit - a passage of the planet in front of its host star. However, atoms in such a tenuous tail have a tendency to relax to their lowest-energy (ground) state. In this state, most atoms absorb mainly ultraviolet light, and measuring such absorption is difficult for two reasons.

First, Earth's atmosphere is opaque to most ultraviolet light, which means that absorption measurements must be made from space. Currently, only Hubble has the capability for ultraviolet studies of exoplanetary atmospheres, and this telescope could reach the end of its mission lifetime in the next decade. Second, the pattern of how much ultraviolet stellar light is absorbed by transiting planets as a function of time or wavelength tends to be complex. Such complexity makes it difficult to interpret ultraviolet measurements of a transiting planet's atmosphere.

Fortunately, helium atoms have a long-lived (metastable) state, in addition to the ground state. Metastable helium atoms absorb nearinfrared stellar light, which has a wavelength only slightly beyond the limits of human vision. Measurements at this wavelength are much easier to interpret than those at ultraviolet wavelengths.

Spake and colleagues observed a transit of WASP-107b, and measured the amount of near-infrared stellar light that was transmitted through the planet's eroding atmosphere as a function of wavelength. The authors identified a narrow absorption feature that they associated with metastable helium atoms (see Fig. 1 of the paper ${ }^{4}$ ). This signal is more than five times greater than any false signal that could be produced by stellar activity.

Detecting helium in the escaping atmospheres of other exoplanets will be difficult because the absorption signal is intrinsically weak, especially for planets smaller than WASP-107b. However, astronomers will eagerly rise to the challenge. The near-infrared signature of metastable helium is readily transmitted through Earth's atmosphere, which means that eroding exoplanetary atmospheres could be probed using ground-based telescopes. The advent of a new generation of extremely large telescopes at ground-based observatories $^{7}$ will allow astronomers to study the escaping atmospheres of planets as small as Neptune, which has a radius four times that of Earth.

Theorists have predicted that the atmospheres of Neptune-sized exoplanets could be rich in helium ${ }^{8}$, owing to differences in the rates at which hydrogen and helium are lost to space. Like other giant planets, these bodies are thought to start out with atmospheres of predominantly hydrogen, abundant helium and smaller amounts of elements heavier than helium. As their atmospheres escape, hydrogen is lost fastest, leading to a gradual relative enrichment in the helium content of the atmosphere.

Heavier elements such as carbon and oxygen would be slow to escape, and could in principle be present in exoplanetary atmospheres in concentrated amounts. These heavier elements are key to understanding both how planets form and how they acquire their atmospheres. For planetary astronomers, an escaping atmosphere that is rich in heavy elements is something of a cosmic treasure, providing ample scientific opportunities to study planetary formation and evolution. Spake and colleagues' detection of helium in WASP-107b will enable astronomers to look for atmospheres that are rich in helium, and perhaps in heavier elements, thereby opening a new subfield of exoplanetary science.

Drake Deming is in the Department of Astronomy, University of Maryland, College Park, Maryland 20742, USA.

e-mail:ddeming@astro.umd.edu

1. Alpher, R. A., Bethe, H. \& Gamow, G. Phys. Rev. 73, 803-804 (1948).

2. Seager, S. \& Sasselov, D. D. Astrophys. J. 537 916-921 (2000).

3. Moutou, C., Coustenis, A., Schneider, J., Queloz, D. \& Mayor, M. Astron. Astrophys. 405, 341-348 (2003).

4. Spake, J. J. et al. Nature 557, 68-70 (2018)

5. Vidal-Madjar, A. et al. Nature 422, 143-146 (2003)

6. Ben-Jaffel, L. \& Ballester, G. E. Astron. Astrophys. 553, A52 (2013).

7. Liske, J., Padovani, P. \& Kissler-Patig, M. Proc. SPIE 8444, 84441I (2012)

8. Hu, R., Seager, S. \& Yung, Y. L. Astrophys. J. 807, 8 (2015).

\title{
PALAEONTOLOGY
}

\section{Evolutionary insights from an ancient bird}

\section{Ichthyornis dispar is a key extinct bird species from when birds were shedding characteristics of their dinosaur ancestors and evolving their current features. A reconstructed skull of I. dispar now illuminates this transition. SEE LETTER P.96}

\section{KEVIN PADIAN}

$\mathrm{T}$ The distinctive features of birds, from beaks to feathers, provide a stark separation between avians and other animal groups. But how did the features of the bird skull evolve? On page 96, Field et al. ${ }^{1}$ present a computerized reconstruction of the skull of a pivotal early bird that brings avian evolution into sharper focus.

In the late 1800s, the palaeontologist Othniel C. Marsh and his field crews made many of the first reported discoveries of ancient dinosaurs and mammals from western North America, amassing a fossilized 'bestiary' that dwarfed what was then known from Europe ${ }^{2}$. Marsh's treasures were constantly in the headlines, perhaps never more so than when he published ${ }^{3}$ his monograph Odontornithes in 1880, which reported several previously undescribed fossil birds of the mid-Cretaceous period (around 80 million to 87 million years ago) from the shores of Kansas and nearby states. Familiar yet strange in many ways, these creatures were so archaic that they retained teeth and substantial bony tails, thus providing clues to the reptilian origin of birds. When Charles Darwin received a copy of the monograph from Marsh, the letter that he wrote back to Marsh said: "Your work on these old birds and on the many fossil animals of N. America has afforded the best support to the theory of evolution, which has appeared within the last 20 years" (see go.nature.com/2hhjxrd).

The specimens Marsh presented in Odontornithes were predominantly from two contrasting bird genera: Hesperornis, which was flightless and essentially wingless, standing 1.3-1.8 metres tall and comparable to today's loons, and a tern-like bird called Ichthyornis, which had an average wingspan of about 60 centimetres (ref. 3). However, neither was closely related to living loons or terns. Both birds had many sharp, curved teeth, which were absent only from the front part of the upper jaw, and their beaks were covered by a horny sheath. Unfortunately, the excavated bones, being small, fragile and of an elaborate architecture, were badly crushed, and proved challenging to prepare. The restoration, mounting and illustration of the specimens were, shall we say, somewhat overenthusiastic. The specimens could be convincingly described only after the mounts had been 
disassembled and prepared afresh more than a century later ${ }^{1,4}$.

Fast forward to the twenty-first century, and in the past 20 years some of the most sensational dinosaur discoveries have been the seemingly endless reports of 'feathered' dinosaurs and newly identified early birds, mainly from Cretaceous deposits in China. These specimens are closer to Archaeopteryx (the earliest known bird, from the Late Jurassic of Germany about 145 million years ago) than to Hesperornis and Ichthyornis ${ }^{5}$. These discoveries have shown that the evolution of feathers, from hair-like down to flight feathers, broadly paralleled the sequence of development of the features of a single feather in living birds ${ }^{6}$. Such insights suggest a plausible sequence for the evolution of wings and flight in birds, whereby newly hatched ancient dinosaurs flapped their incipient wings as a way of boosting their ability to scale steep inclines when evading predators ${ }^{7}$.

But many questions persist about the anatomical changes in early bird evolution, and this is where the work of Field and colleagues comes in. Present-day birds have skulls that are different in many ways from those of all other animals, including the dinosaurs from which they evolved. Bird snouts are lightweight, usually narrow and sometimes quite long. Indeed, the bones of the bird snout are relatively light and fragile compared with those of other animals, and these structures are covered by a strong beak made of the protein keratin, which enables birds to access various foods, such as seeds or carcasses. Inside the beak is a complex of bones that corresponds to the human palate; but unlike ours, the bird bones have mobile connections to each other and to the surrounding skull and jaw bones. This system of mobility is an elaboration of the basic dinosaurian one, and is key to accommodating the diverse feeding habits of birds.

Moreover, 'bird brain' is not the insult you might think. Bird brains are larger relative to their body size than is the case for reptiles, and the relative size of bird brains is comparable to that of placental mammals. As birds evolved from their dinosaur ancestors, the bones that protect the brain enlarged to keep pace with the changes in brain size. The bones of the skull roof and cheek region are also comparatively larger than the equivalent structures in their dinosaur ancestors, whereas the adductor muscles of the bird jaw are reduced. But in what order did these features evolve, and how did they shape avian evolution?

Ichthyornis is closely related to living birds, but retains many features of the earliest birds. No Ichthyornis skull material had been uncovered since Marsh's discoveries in the 1870s. But Field et al. describe four new threedimensionally preserved specimens with skull remains, and they image them in $3 \mathrm{D}$, along with some overlooked skull bones from Marsh's original specimens. The authors used a standard technique called high-resolution computed

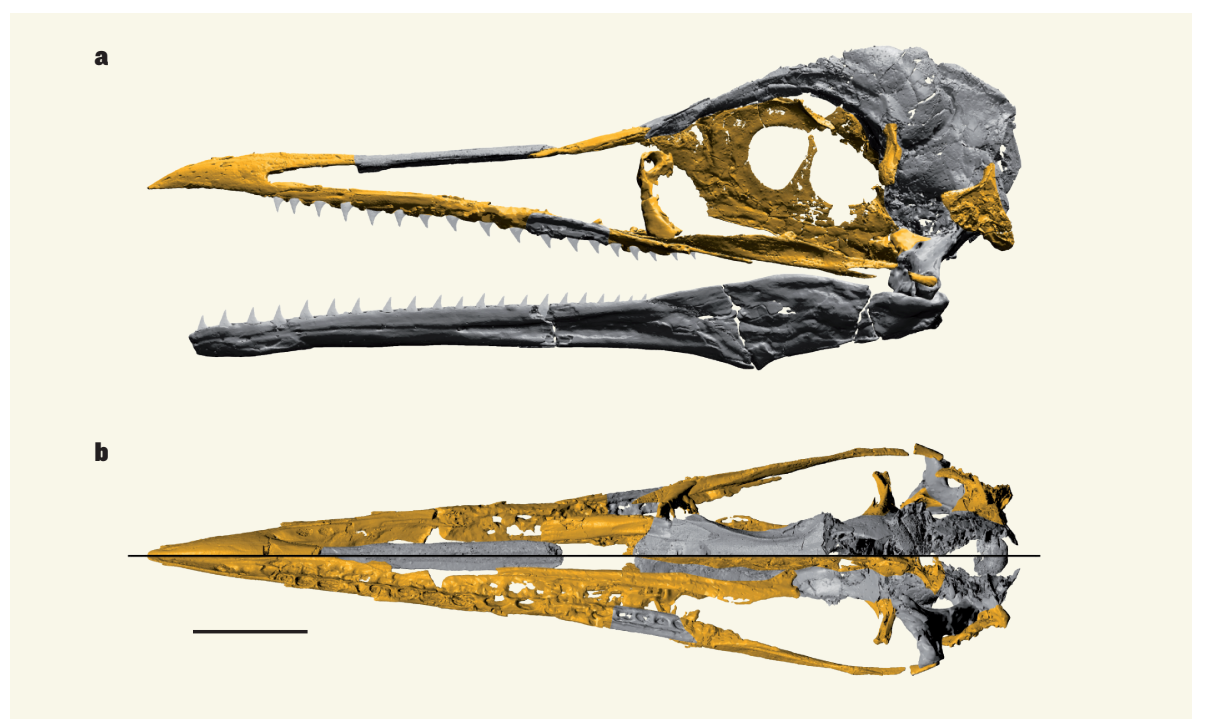

Figure 1 | Skull of the bird Ichthyornis dispar. Field et al. ${ }^{1}$ report the reconstruction of the skull of an extinct species. Their reconstruction fills in some structures missing from previously available fossils, thereby illuminating the transition between the loss of ancient dinosaur features and the evolution of characteristics found in present-day birds. The sections in yellow are newly identified fossil material, whereas the grey structures have been described previously. a, A side view of the skull. b, A view from above the skull (beak positioned on the left) showing cross-sections in two focal planes. The section above the black line is closer to the top of the skull than the region below the black line. Scale bar, 1 centimetre. (Adapted from Extended Data Fig. 2 of ref. 1.)

tomography, in which a reconstruction of each bone is compiled by taking extremely thin cross-sectional images all the way through the bone, like slicing a salami sausage and reassembling it. This enables the internal anatomy and outer shape to be visualized. The images of all the separate bones are assembled, and a computer program enables the bone images to be manipulated, allowing analysis of how the bones might have moved.

The resulting skull images (Fig. 1) show that the beak of Ichthyornis has some features that place it between the earliest birds and living birds: the beak was small, had not yet evolved a bony shelf structure in the palate and was limited to the tip of the jaw. However, the probable mobility of the Ichthyornis skull seems to be more like that of living birds. The brain would have been much like those of today's birds, but the cheek region, bounded by bones of the skull roof and the side of the skull, has characteristics that are closer to those of dinosaurs, such as the retention of a large bony chamber for the adductor muscles that close the jaw. Therefore, several key features of the brain and palate evolved before the jaw muscles became reduced and the familiar features of the beak of living birds evolved.

This study raises many questions that remain to be answered. For example, were there functional changes that went along with reducing the jaw muscles from the ancestral dinosaurian condition? Did this change reflect a change in diet? And what ecological habits are correlated with the loss of teeth from the front part of the upper jaw and the evolution of the horny beak that covers it? Hesperornis was probably a diver that hunted fishes and invertebrates in the water column, whereas Ichthyornis seems to have been more a surface skimmer or perhaps a shallow plunger like a tern or gull ${ }^{2-4}$. How did these different predatory approaches favour the same pattern of tooth reduction, which also happened independently in other early bird groups? How did the mobility of the bones of the palate against adjacent skull bones in Ichthyornis compare with the ranges of motion in the palates of dinosaurs and living birds, and what might these evolutionary changes suggest about the diet and mode of feeding of Ichthyornis?

Whatever the answers to these questions turn out to be, Field and colleagues' beautifully rendered 3D scans and reconstructions of this iconic fossil avian, along with their comparisons of these structures with those of earlier and later birds, provide an important resource to aid our understanding of early bird evolution.

Kevin Padian is in the Department of Integrative Biology and the Museum of Paleontology, University of California, Berkeley, Berkeley, California 94720, USA. e-mail:kpadian@berkeley.edu

1. Field, D. J. et al. Nature 557, 96-100 (2018).

2. Plate, R. The Dinosaur Hunters (McKay, 1964)

3. Marsh, O. C. Odontornithes: A Monograph on the Extinct Toothed Birds of North America (US Govt Printing Office, 1880)

4. Clarke, J. A. Bull. Am. Mus. Nat. Hist. 286, 1-179 (2004).

5. Chiappe, L. M. \& Meng, Q. Birds of Stone: Chinese Avian Fossils from the Age of Dinosaurs (Johns Hopkins Univ. Press, 2016).

6. Prum, R. O. \& Brush, A. H. Sci. Am. 288, 84-93 (2003).

7. Dial, K. P. Science 299, 402-404 (2003). 